\title{
THE DIGITIZED SECOND PALOMAR OBSERVATORY SKY SURVEY (DPOSS). II. PHOTOMETRIC CALIBRATION
}

\author{
R. R. Gal, ${ }^{1}$ R. R. de Carvalho, ${ }^{2}$ S. C. Odewahn, ${ }^{3}$ S. G. Djorgoviski, A. Mahabal, \\ R. J. BRunNer, ${ }^{4}$ AND P. A. A. LOPES ${ }^{5}$ \\ Palomar Observatory, Mail Code 105-24, California Institute of Technology, Pasadena, CA 91125 \\ Received 2001 November 25; accepted 2002 September 24
}

\begin{abstract}
We present the photometric calibration technique for the Digitized Second Palomar Observatory Sky Survey, used to create seamless catalogs of calibrated objects over large sky areas. After applying a correction for telescope vignetting, the extensive plate overlap regions are used to transform sets of plates onto a common instrumental photometric system. Photometric transformations to the Gunn gri system for each plate, for stars and galaxies, are derived using these contiguous stitched areas and an extensive CCD imaging library obtained for this purpose. We discuss the resulting photometric accuracy, survey depth, and possible systematic errors.

Key words: catalogs — surveys — techniques: photometric

Online material: color figures
\end{abstract}

\section{INTRODUCTION}

The answers to many important cosmological questions require large sky surveys, encompassing hundreds or thousands of square degrees. Large-scale structure in the universe, the power spectrum at large angular scales, and the distribution of stars in our own galaxy all rely on homogeneous, moderately deep imaging with small (and well-understood) systematic errors, as well as enormous sky coverage. Until very recently, such data was available only from photographic plates, which, with their large physical size, fill the focal plane of Schmidt telescopes. Unfortunately, these plates are notoriously difficult to calibrate, with large sensitivity variations among plates and even within a given plate. Nevertheless, they provide the only current source of all-sky imaging data. Various projects, such as the Automated Plate Scanner (APS; Odewahn \& Aldering 1995, hereafter OA95), have used digitized scans of the first Palomar Sky Survey to generate large-area catalogs. The more recent efforts by the Automatic Plate Measuring team (APM; Maddox et al. 1990, hereafter MES) and other groups have used the Southern Sky Survey plates or scans of the more recent Second Palomar Sky Survey for this purpose. Some of these projects have used only a fraction of the available data, with limited CCD calibration, which makes assessing large-scale photometric errors difficult, if not impossible. For instance, MES used a scheme to transform all of their plates onto a common photometric system but only had CCD calibration data for about one-third of their plates. In the northern sky, the GSC-II (McLean et al. 1998) and the USNO (Monet 1998) both provide catalogs (based on some of the same plate material), but they lack accurate photometric calibration and do not target faint

\footnotetext{
${ }^{1}$ Current address: Department of Physics, University of California, Davis, 1 Shields Avenue, Davis, CA 95616; gal@physics.ucdavis.edu.

2 Current address: Instituto Nacional de Pesquisas Espaciais, Avenue dos Astronautas 1758, São José dos Campos, 12227-010 São Paulo, SP, Brazil.

${ }^{3}$ Current address: McDonald Observatory, University of Texas at Austin, Austin, TX 78712

${ }^{4}$ Current address: Department of Astronomy, University of Illinois, Urbana, IL 61801.

${ }^{5}$ Current address: Observatorio Nacional, Rua General José Cristino 77, 20921-400 Rio de Janeiro, RJ, Brazil.
}

objects. Only the recently begun Sloan Digital Sky Survey (SDSS; York et al. 2000) will provide improved data in the northern sky and only at Galactic latitudes $|b|>40^{\circ}$.

To address these issues and provide the community with a large catalog of sources with accurate photometry, we have produced the Digitized Second Palomar Observatory Sky Survey (Djorgovski et al. 1998, 2003). Prior papers have discussed the object detection and classification techniques using the SKICAT software package (Weir et al. 1995b, 1995c), and a companion paper (Odewahn et al. 2004) presents more recent artificial neural network (ANN) and decision tree (DT) classifiers employed in this survey. Gal et al. (2000a) presented the vast CCD imaging sample obtained at the Palomar 60 inch $(1.5 \mathrm{~m})$ for the purpose of calibrating DPOSS. Here we discuss the techniques used to derive the plate photometric calibration and generate a seamless catalog of objects over the high Galactic latitude $\left(|b|>30^{\circ}\right)$ northern sky. We briefly review the salient details of the DPOSS and CCD object detection and photometry schemes in $\S 2$. The use of plate overlap regions to transform sets of plates on a uniform instrumental magnitude system is described in $\S 3$. The derivation of the photometric calibration using CCD data is discussed in $\S 4$, including our final photometric errors. We conclude with a brief discussion of potential applications and future developments in $\S 5$. We note that this paper supersedes the earlier discussion of DPOSS calibration presented in Weir et al. (1995a).

\section{DPOSS AND CCD DATA}

\subsection{DPOSS}

The POSS-II photographic survey (Reid et al. 1991) covers the entire northern sky $\left(\delta>-3^{\circ}\right)$ with 897 overlapping fields (each 6.5 , with $5^{\circ}$ spacings) and, unlike the old POSS-I, has no gaps in the coverage. Approximately half of the survey area is covered at least twice in each band, because of plate overlaps. Plates were taken at the Palomar 48 inch $(1.2 \mathrm{~m})$ Oschin Schmidt telescope in three bands: blue-green, IIIa-J+GG395, $\lambda_{\text {eff }} \sim 480 \mathrm{~nm}$; red, IIIa-F + RG610, $\lambda_{\text {eff }} \sim 650 \mathrm{~nm}$; and very near IR, IV-N + RG9, $\lambda_{\text {eff }} \sim 850 \mathrm{~nm}$. The bandpasses are illustrated in Figure 1. Typical limiting magnitudes reached are $g_{J} \sim 21.5, r_{F} \sim 21.0$, and $i_{N} \sim 20.3$, i.e., $\sim 1-1.5$ mag deeper 


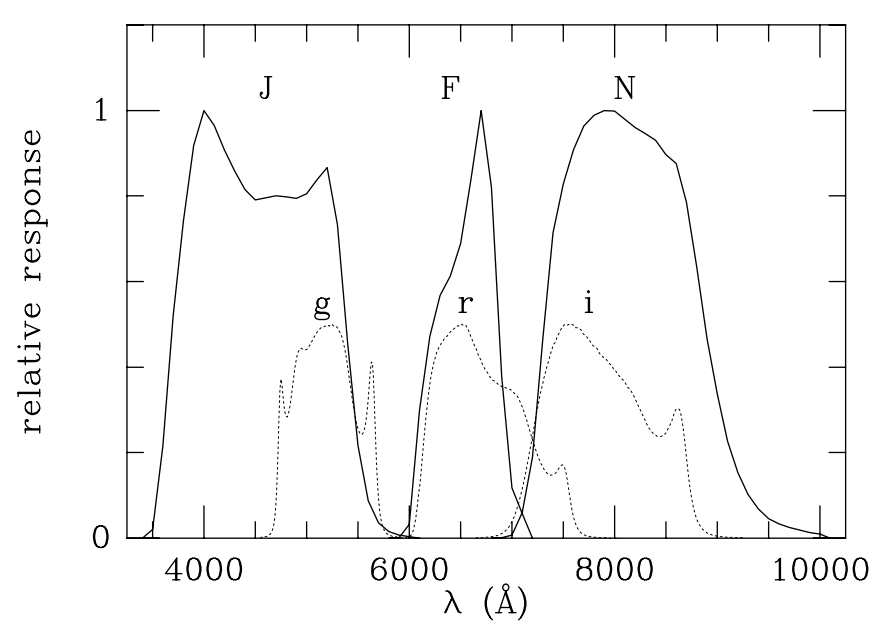

FIG. 1.-POSS-II JFN (solid lines) and Gunn gri (dashed lines) bandpasses.

than the POSS-I. The image quality is improved relative to the POSS-I and is comparable to the southern photographic sky surveys.

The original survey plates are digitized at the Space Telescope Science Institute (STScI), using modified PDS scanners (Lasker et al. 1996). The plates are scanned with $15 \mu \mathrm{m}\left(1^{\prime \prime} 0\right)$ pixels, in rasters of 23,040 square, giving $\sim 1$ Gbyte plate $^{-1}$, or $\sim 3$ Tbytes of pixel data total for the entire digital survey (DPOSS). The preliminary astrometric solutions provided by GSC-II (McLean et al. 1998) are good to rms $\sim 0^{\prime \prime} 5$ and are in the process of being improved substantially.

An extensive effort, centered at Caltech and with sites in Italy (Osservatorio Astronomico di Capodimonte and Osservatorio Astronomico di Roma) and Brazil (Observatório Nacional), has resulted in the processing, calibration, and cataloging of nearly all scans at $|b|>10^{\circ}$, with the detection of all objects down to the survey limit and star/galaxy classifications accurate to $90 \%$ or better down to $\sim 1 \mathrm{mag}$ above the detection limit. Object detection and photometry is performed by SKICAT, a novel software system developed for this purpose (Weir et al. 1995a). It incorporates some standard astronomical image processing packages, a commercial Sybase database, as well as a number of artificial intelligence (AI) and machine-learning (ML) modules. We measure $\sim 60$ attributes per object on each plate in each filter. A subset of these are used for classification, as described in Odewahn et al. (2004).

\subsubsection{Vignetting Correction}

Large-area detectors have always faced the problem of nonuniform illumination across the detector. The resulting effect, primarily due to placing a square detector in a telescope with a round aperture, is called vignetting. The vignetting pattern must be removed if one is to use the data at larger radii from the plate center. A simple (but incorrect) approach is to assume a circularly symmetric function and apply it to the data. However, although largely radially symmetric, the pattern has finer structure due to the bending of the plates, the filters used, etc. Here we describe the procedure we have used to remove the vignetting pattern from the DPOSS data. This procedure is performed separately for the three filters used.

Basically, we stack and combine multiple DPOSS plate images to obtain a "master vignetting correction" field, in a manner analogous to generating a flat field for CCD data. Because this procedure was developed after most plates were processed into catalogs, the vignetting correction image is used to derive corrections to the object magnitudes postprocessing, as well as to correct the actual pixel data. The following procedure was followed, using 100 plates for each band:

1. Bin each plate image $8 \times 8$, resulting in a $2880 \times$ 2880 pixel image.

2. Normalize each image by the median of its central $720 \times$ 720 region.

3. Stack the resulting images. This results in a $2800 \times$ $2800 \times 100$ array.

4. For each pixel in the array, discard the 10 brightest and 10 faintest members and obtain the median of the remaining 80 . This produces a single $2880 \times 2880$ image, where each pixel is now a clipped median of the 100 contributing images.

5. Normalize the resulting image such that the maximum in the central $720 \times 720$ pixel region is 1.0 .

Thus, all corrections are relative to the plate center, which is the best-exposed part of the plate. Contours of the master vignetting correction image in the $F$ and $J$ bands are shown in Figure 2; the $N$ map is similar.

Nearly all plates at $|b|>10^{\circ}$ have been processed into catalogs. The final result of this processing is the Palomar-Norris Sky Catalog (PNSC), expected to contain $\sim 50 \times 10^{6}$ galaxies and more than $2 \times 10^{6}$ stars.

\subsection{CCD Data}

Details of the acquisition, processing, and calibration of the CCD data used to calibrate DPOSS are provided in Gal et al. (2000a). Here we provide a brief overview of these data.

We have imaged nearly 900 independent CCD fields for DPOSS calibration. The imaging targets are selected from the list of northern Abell clusters (Abell et al. 1989), with priority given to the richest clusters closest to the field centers. This strategy increases the number of objects for both star-galaxy separation and photometric calibration. For those plates with no Abell clusters, we image the plate center and two other pointings within the plate. All data are obtained at the Palomar Observatory 60 inch telescope with the CCD imaging cameras placed at Cassegrain focus. The Gunn gri filters (Thuan \& Gunn 1976; Wade et al. 1979; Schneider et al. 1983) are used, which are well matched to the DPOSS bandpasses (see Fig. 1). Data are taken only on photometric nights with seeing better than $2^{\prime \prime}$. The mean seeing for our data is $\sim 1 " .5$, with limiting magnitudes of approximately $m_{\mathrm{lim}}=21.5,21.5$, and $21.2 \mathrm{mag}$ in $g, r$, and $i$, respectively $(\sim 0.5-1$ mag deeper than the plate detection limits). For every night that is deemed photometric, we observe a set of Gunn standards (Kent 1985).

Data are processed in the usual way using the IRAF data reduction package (Tody 1986). All frames are bias-subtracted and flat-fielded, using a combination of dome and twilight flats. In addition, a dark sky flat-field correction is generated by median-filtering all of the unregistered target frames in each filter on a given night. Finally, we apply a fringe correction to the $i$ images. The photometric standard stars observed on each night are photometered using the APPHOT package in IRAF, and the resulting collection of between five and 12 measured instrumental magnitudes is used to determine the zero-point offset, air-mass term, and color term in each filter.

Object detection on the target frames is performed using the FOCAS package (Jarvis \& Tyson 1981; Valdes 1982). The $g$, $r$, and $i$ frames are processed independently, using detection parameters of $2.5 \sigma$ pixel $^{-1}$, a 25 pixel minimum area, and a sky value estimated individually for each image. Object classification is also performed by FOCAS, and the classifications 

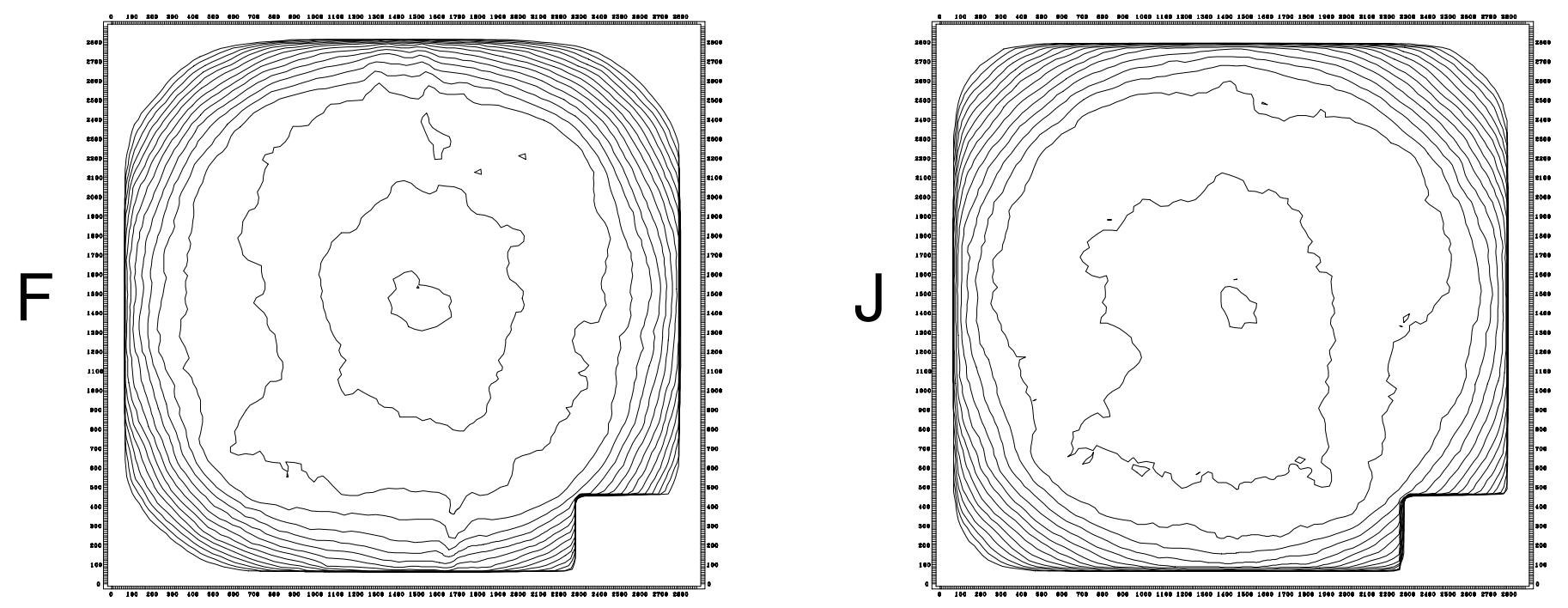

FIG. 2.-Contour maps of the vignetting correction function for the $F$ and $J$ bands.

are visually inspected. Bright objects with incorrect classifications (usually due to saturated pixels) are corrected by hand. The photometric coefficients (zero-point, color term, and airmass term) derived from the standard-star observations are used to determine object magnitudes.

\section{PLATE OVERLAP ANALYSIS}

A great advantage of POSS-II over earlier surveys is the extensive overlap between neighboring plates. Adjacent plates overlap each other by 1.5 along edges covering 6.5 , providing $\sim 9 \mathrm{deg}^{2}$ of duplicated area for each plate pair. This compares favorably with the APM survey, whose scans typically had $1^{\circ}$ overlaps (Maddox et al. 1990); with the strong effect of vignetting at plate edges, the extra $0^{\circ} .5$ of overlap in DPOSS is significant. These areas each contain thousands of objects, which can be used to derive the transformation of one plate's instrumental magnitude system to that of its neighbor. These transformations can be propagated across multiple boundaries to tie a contiguous set of plates onto a common photometric system.

In practice, this instrumental magnitude transformation must be mapped independently for stars and galaxies, especially for brighter magnitudes $\left(m_{\text {inst }}<19\right)$. Although the density-tointensity (D-I) transformation has been linearized using the densitometry spots on each plate, stars and galaxies nevertheless display distinct photometric behaviors. This is because the brighter pixels in stellar images populate the highly nonlinear part of the D-I relation, where small errors in the polynomial fit (mostly due to the small number of points characterizing the "shoulder" of the curve) result in larger variations in the derived intensity. Pixels within galaxies occupy the more linear part of the D-I transformation, resulting in more stable and linear photometry.

In addition, propagating the photometric transformations across a large number of plates (as would be necessary to knit the whole sky) can result in the unacceptable accumulation of (possibly systematic) errors. This issue was addressed by Groth \& Peebles (1986), and their technique was used by MES to estimate the extent of such errors in the APM. A similar approach was also taken by OA95 in an analysis of galaxy counts at the north Galactic pole, but that study was limited by the small overlap regions and poorer quality of POSS-I. An early attempt to address the problem of plate inhomogeneities in POSS-II is discussed in Picard (1991).

To avoid this issue entirely, we have elected to calibrate each plate using a sliding boxcar technique, described below. We transform each plate's eight surrounding neighbors onto the instrumental system of the center plate, match all the CCD data available for the resulting nine-plate area, and derive the photometric calibration for the center plate. The individually calibrated plates can then be quilted with no additional offsets, because they are already on a calibrated, rather than instrumental, magnitude system. Unlike MES and OA95, we are allowed this luxury by the large quantity of available CCD calibration data. Figure 3 illustrates the processed DPOSS plates (squares) and the available CCD data (dots).

First, we determine which plate pairs have overlap regions larger than $\sim 5 \mathrm{deg}^{2}$, which removes pairs where only the heavily vignetted corners overlap. The overlap regions are matched, and the outermost $10 \%$ of the matched area is clipped on all four sides to avoid the most heavily vignetted regions. A spline is fitted to the magnitude difference of the matched objects from the two plates, as a function of instrumental magnitude, $\Delta m=m_{\text {plate } 1}-m_{\text {plate } 2}=f\left(m_{\text {plate } 1}\right)$. This fit is performed independently for stars and galaxies in each filter $(J F N)$. At faint magnitudes $\left(m_{\text {inst }}>19.0\right)$ we use both stars and galaxies in the independent fits to avoid biases due to possible differences in classification between plates. At these faint magnitudes there are no bright pixels (which populate the highly nonlinear part of the D-I relation) in the stellar images.

There are typically between 5000 and 30,000 objects used to determine the transformation between a given plate pair. Although significant scatter is present, especially at faint magnitudes, the mean is nevertheless well determined. At the bright end $(m<16)$, where the number of objects is small and classification problems exist, points for the spline fit are placed by hand. At intermediate magnitudes (typically $16<m<20$ ), a robust locus-mapping algorithm is used. Finally, at the faint end $(m>20)$, where incompleteness is a factor, the fit is fixed at a constant $\Delta m$. These objects are in any case too faint to be photometered and classified properly, and they are therefore of limited scientific use from the current survey. Figure 4 shows a typical pair of plate overlaps for galaxies (top) and 


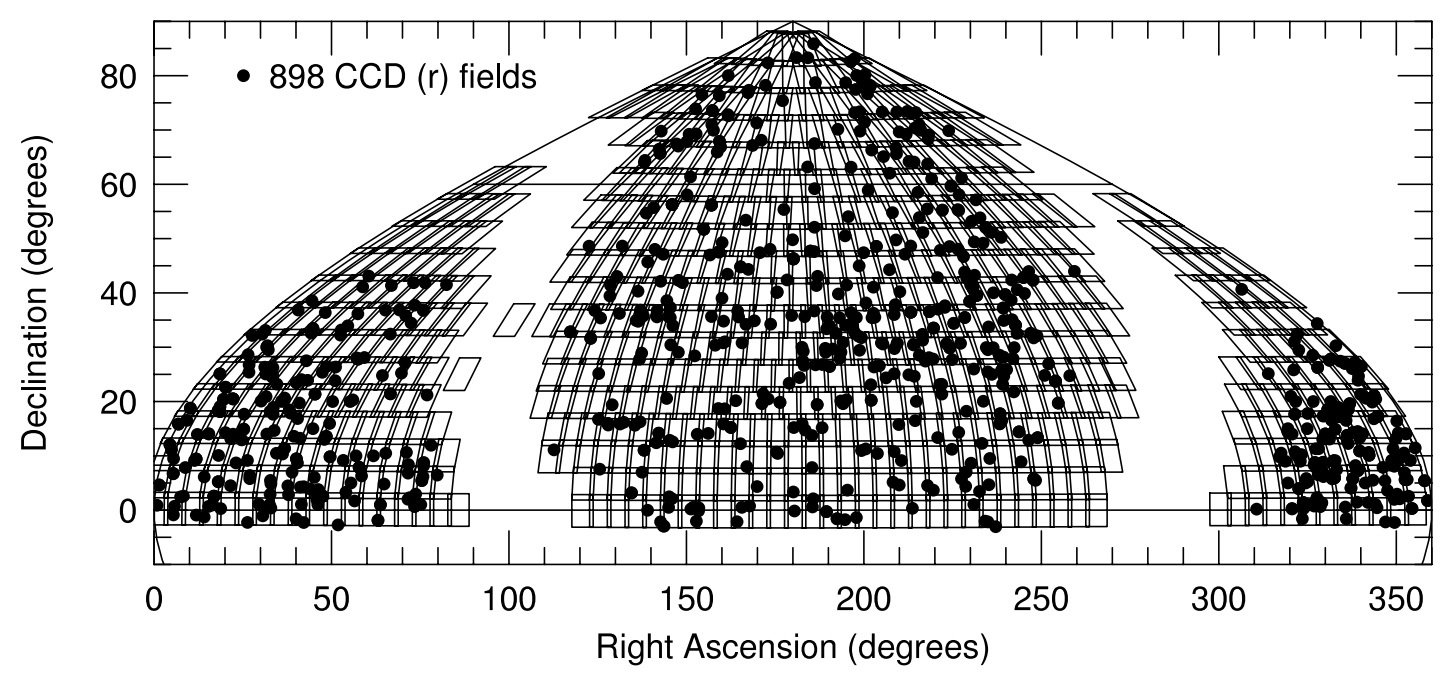

FIG. 3.-Processed DPOSS plates (squares) and available CCD calibration pointings (dots).

stars (bottom) matched between plates 392 and 333. The resulting spline fits are also shown.

In contrast, MES defined a single coefficient, $T_{i j}$, to match the magnitudes between adjacent plates. While they state that the rms of a linear fit to the magnitude differences [in their case, $\left(m_{i}-m_{j}\right)$ vs. $\left.\left(m_{i}+m_{j}\right) / 2\right]$ is nearly as good as a higher order polynomial, we find that this is not always the case and is certainly not true for stars (which they do not treat). We therefore have elected to use our procedure as described above, which is more general but requires a great deal more interaction
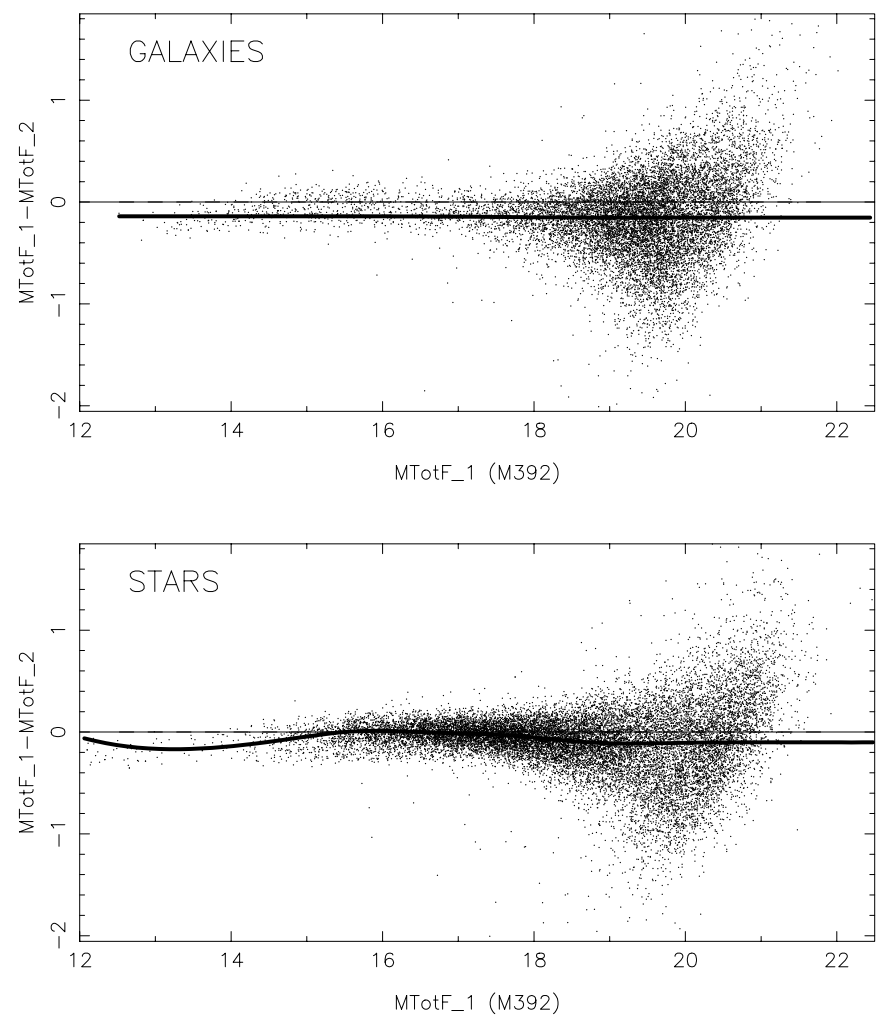

FIG. 4.-Typical plate overlap analysis, for $F$ plates in fields M333 (cat2) and M392 (cat1). We show the magnitude difference between plates for galaxies (top) and stars (bottom), along with the spline fits to unify the photometric systems. Brighter than $m=16$, the galaxy sample is heavily contaminated by misclassified stars, so the spline is constrained manually. and inspection. This process also permits us to find individual plates that behave abnormally, because of either their intrinsic properties or processing failures.

To actually transform a given plate's instrumental system to that of the reference plate for a given area, we propagate magnitude transformations through sets of up to five plate overlaps connecting the plate being transformed to the fiducial plate. This is illustrated in Figure 5, where arrows represent the transformation paths used to tie plates 392 and 452 to the center (fiducial) plate 391. A plate directly adjacent to the center plate can be tied to it by up to four paths (solid arrows), while a plate diagonally away from the center can be tied by up to five paths (dashed arrows). The five plate limit is imposed to avoid propagating errors across a large number of plate overlaps. Figure 6 shows the five photometric transformations derived between plates 331 and 391. The ordinate shows the fiducial plate magnitude $m_{f}$, while the abscissa shows the difference between the magnitude on the plate to be transformed, $m_{p}$, and $m_{f}$. Rather satisfyingly, all possible convolution paths

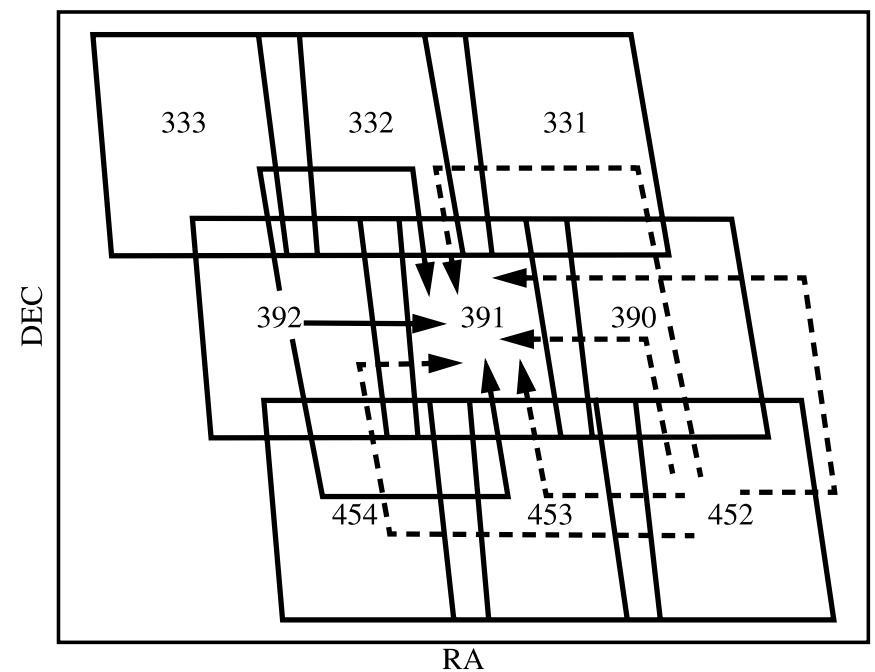

FIG. 5.-Plate overlaps used to derive mean instrumental photometric transformations. Arrows represent the transformation paths used to tie fields 392 and 452 to the center (fiducial) plate 391. A plate directly adjacent to the center plate can be tied to it by up to three paths (solid arrows), while a plate diagonally away from the center can be tied by four paths (dashed arrows). 

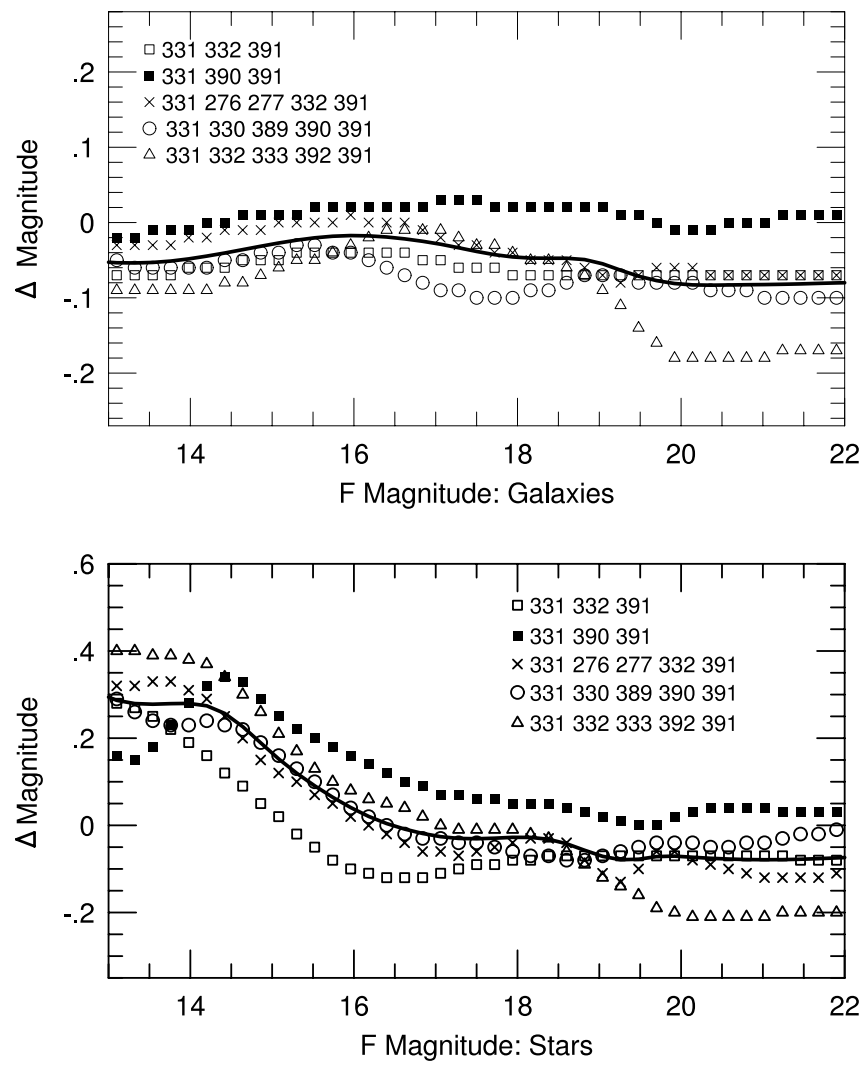

FIG. 6.-Results of the overlap analysis illustrated in Fig. 4. The ordinate shows the fiducial plate magnitude $m_{f}$, the abscissa shows the difference between the magnitude on the plate to be transformed $m_{p}$ and $m_{f}$. Rather satisfyingly, all possible transformation paths provide similar transformations for a given plate. provide similar transformations for a given plate, especially for galaxies; the rms of the differences of each path from the mean reflect the scale of photometric errors due to plate pistoning. These transformations are averaged for each plate to generate a spline $\Delta m_{p f}=m_{p}-m_{f}$ as a function of $m_{f}$, shown as the solid line in Figure 6. These plots are visually inspected for each plate pair, and instances of abnormal behavior, such as highly variable transformations, are flagged and treated by hand. This technique allows us to detect plate overlaps with unusual behaviors and provides more stable magnitude matching than is possible using a single overlap. The transformations for stars are highly nonlinear and show much more variance than those for galaxies. This contributes to the large photometric errors in stellar photometric calibration discussed in $\S 5$. Figure 7 demonstrates the removal of plate-to-plate pistoning using this technique. The left panel shows galaxies in an uncorrected instrumental magnitude range of $0.5 \mathrm{mag}$, from an area covering 15 plates. Individual plates are evident as square patches of varying galaxy density. The right panel shows galaxies from the same area after the instrumental magnitudes have been homogenized; individual plates are no longer discernible.

\section{CALIBRATION TO THE GUNN gri SYSTEM}

Once a set of nine plates has been transformed to a common instrumental system (that of the center plate), we match our CCD catalogs to the plate catalogs for that area. Typically, we have between 15 and 50 CCD pointings in the three filters (gri) for a given nine plate area. This provides between 8000 and 30,000 matched galaxies and a similar number of stars from which to derive photometric calibration.

The CCD and plate data are matched, allowing for an overall offset, scale change, and rotation between the CCD-derived celestial coordinates and the plate celestial coordinates. After the mean CCD-plate coordinate offset is derived, a search radius of $3^{\prime \prime}$ is used, and the search process iterated until the right ascension and declination offsets converge. Typical coordinate residuals are 0.4 , which both confirms the stated astrometric
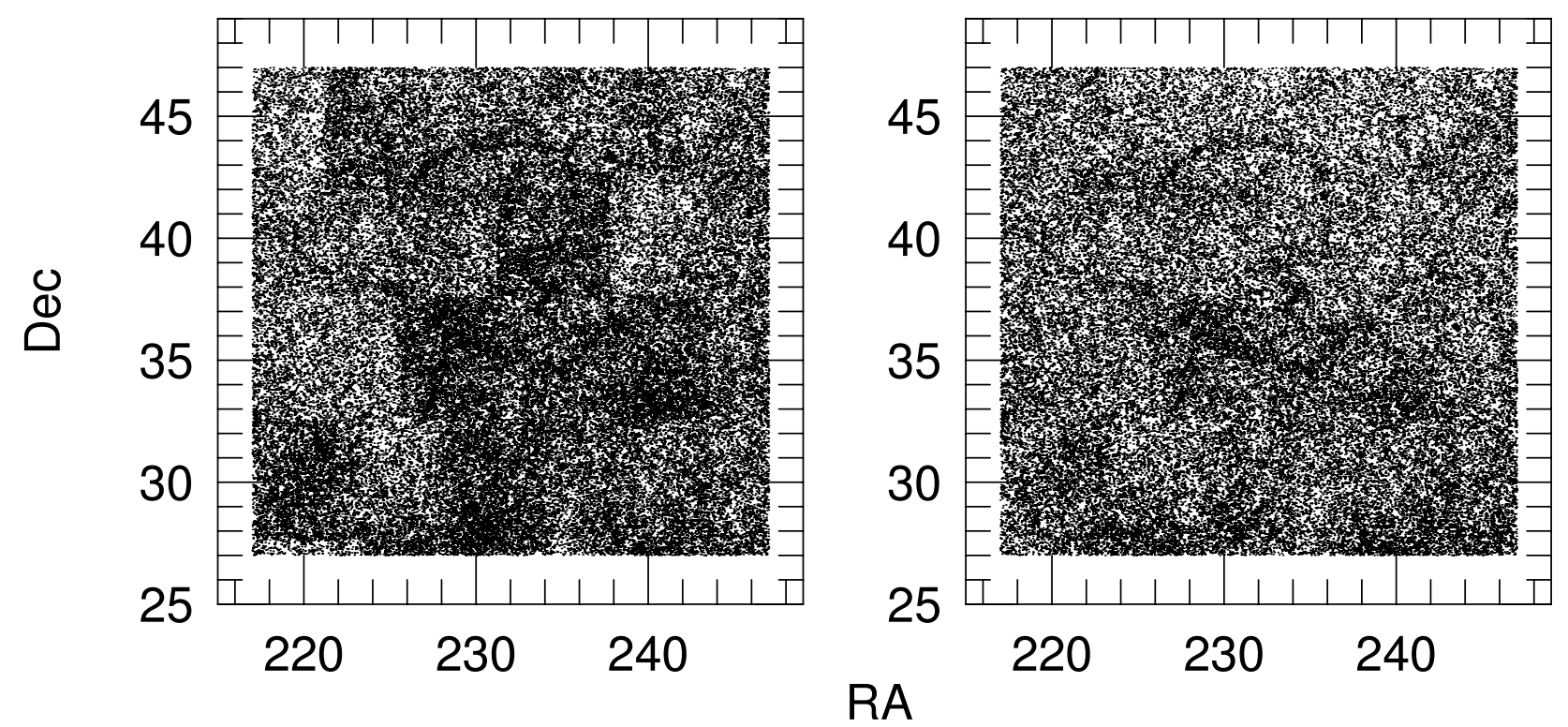

FIG. 7.-Galaxies from a set of 15 plates before and after removal of plate pistoning. The left panel shows galaxies in an uncorrected instrumental magnitude

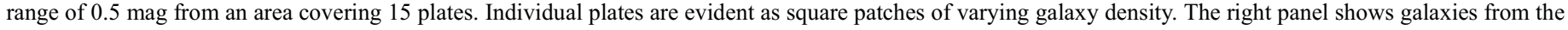
same area after the instrumental magnitudes have been homogenized; individual plates are no longer discernible. 

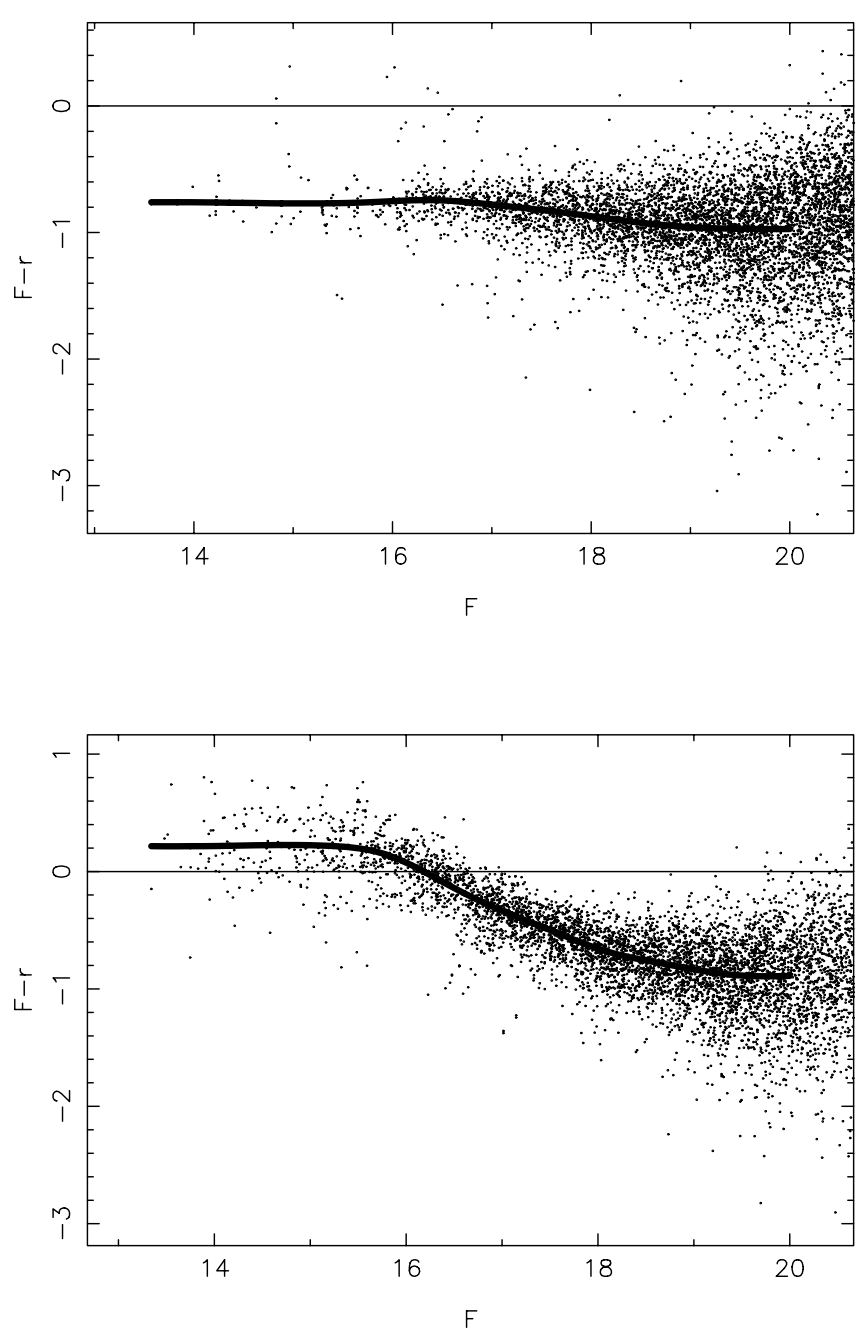

FIG. 8.-Transformation from DPOSS $F$ magnitudes to Gunn $r$ magnitudes derived for a set of nine plates, for galaxies (top) and stars (bottom). A linear fit, much less a single zero-point offset, is clearly insufficient.

accuracy of DPOSS and implies that false matches are not a significant source of added noise. A spline is fitted to the difference between the CCD calibrated magnitude $m_{\mathrm{CCD}}$ and the plate instrumental magnitude $m_{\text {inst }}$ in a manner identical to the plate overlap analysis. Once again, this is done independently for stars and galaxies, relying on the plate-based classification (as this classification ultimately determines which calibration will be applied). Figure 8 shows a typical set of points and the resulting transformations between plate $F$ and CCD $r$ magnitudes, for both stars and galaxies, in a nine plate area. A linear fit is also shown; the nonlinear spline fits are clearly necessary. The derived transformations from $J \rightarrow g$, $F \rightarrow r$, and $N \rightarrow i$ are then applied solely to the center field (391 in the example here).

This method differs sharply from that employed by MES, who obtained only magnitude zero points from their CCD data. Their frames, at $2^{\prime} \times 3^{\prime}$ in size, cover only $6 \%$ of the area of our CCD images, providing a factor of 17 fewer calibrating objects from detector area alone; considering our deeper exposures and more numerous CCD fields (nearly 900 vs. 69), we have a factor of approximately 1000 more calibration objects. The nonlinearities in the transformations to calibrated magnitudes, if not treated, can lead to incorrect number-count slopes and correlation functions.

\subsection{Color Terms}

An examination of the filter curves in Figure 1 shows that the $F \rightarrow r$ and $N \rightarrow i$ transformations are likely to be free of color terms, as the plate and CCD filter systems are well matched. However, the $J$ filter is significantly broader and bluer than the corresponding CCD $g$ filter, and we may expect a color correction to be necessary. In the left panels of Figure 9, we show the difference between calibrated plate magnitude and CCD magnitude, $g_{0}(J)-g_{\mathrm{CCD}}$, as a function of $g-r$ color, for both galaxies and stars. A strong color term is evident, especially for the stars. The galaxies do not span a significant range in $g-r$, making the color term impossible to discern in the noise. Nevertheless, this color correction is extremely important for astrophysical purposes, such as photometric redshift estimation, galaxy typing, and cluster detection.

To estimate the color correction, we calculated the median $g_{0}(J)-g_{\mathrm{CCD}}$ in bins of $\Delta(g-r)=0.1$, shown as filled squares in Figure 9. We fitted a linear relation to these points, resulting in a corrected magnitude,

$$
g_{\text {corr }}(J)=g_{0}(J)-0.373\left[g_{0}(J)-r_{0}(F)\right]+0.177 \text {. }
$$

We apply this correction to both stars and galaxies, with the results shown in the right panels of Figure 9. The correction both removes the slope and reduces the scatter in the relations, as expected.

Inspection of the corresponding data for $r(F)$ and $i(N)$ shows that no color terms are needed for those filters, as expected from the well-matched filters for the plates and CCDs.

\section{PHOTOMETRIC ACCURACY}

Photometric errors in a plate-based survey consist of random errors within plates, zero-point variations within a plate, and plate-to-plate zero-point offsets, also called pistoning. Random errors are due simply to Poisson noise. Zero-point variations within individual plates are due both to telescope vignetting and inhomogeneous emulsion sensitivity. Plate pistoning is due to both varying emulsions between plate batches and differences in observing conditions, such as moonlight and seeing. Using both our extensive CCD calibration set and the large plate overlap regions, we can measure the magnitude of these effects.

\subsection{Comparison with CCD Data}

First, we compare the calibrated plate magnitudes from a large number of plates with the corresponding CCD data. The errors measured in this procedure will include random plate photometric errors, plate-to-plate pistoning, as well as random errors from the CCD photometry. Using the internal tests of the CCD data presented in Gal et al. (2000a), the tests in this section, and the plate overlap tests from the next section, we can decompose our measured errors into the constituent sources.

We plot the plate versus CCD magnitudes in Figure 10. The $1 \sigma$ errors for galaxies are $0.21,0.19$, and 0.32 mag at $m=19.0$ in the $g, r$, and $i$ filters, respectively. Table 1 provides the average photometric errors for galaxies and stars as a function of magnitude for the three bandpasses; these are plotted in Figure 11.

This scatter is an accurate reflection of our accumulated errors. Because the CCD data cover hundreds of plates and come from hundreds of nights, all sources of error are present in this calibration. This includes errors in the zero points of the 

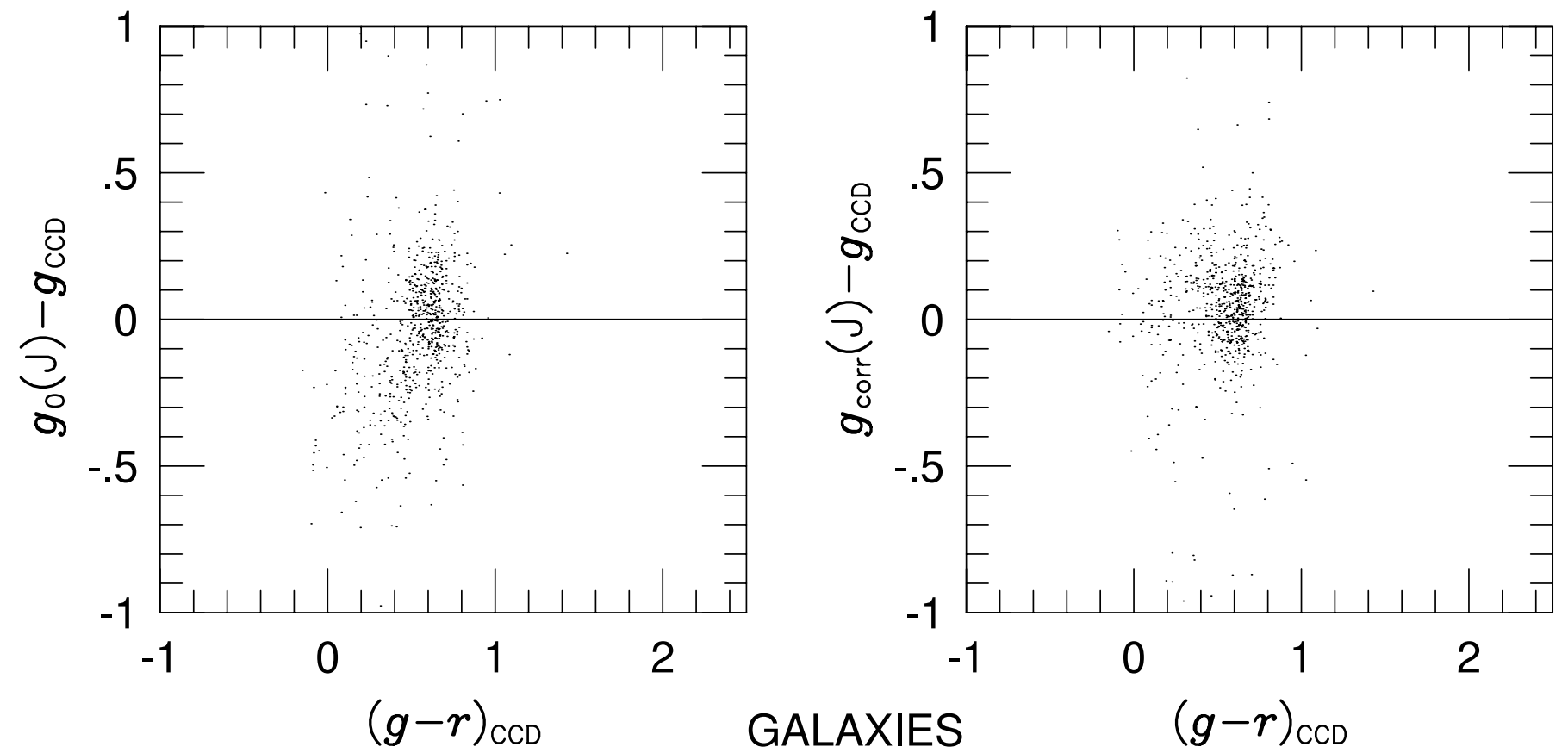

BEFORE
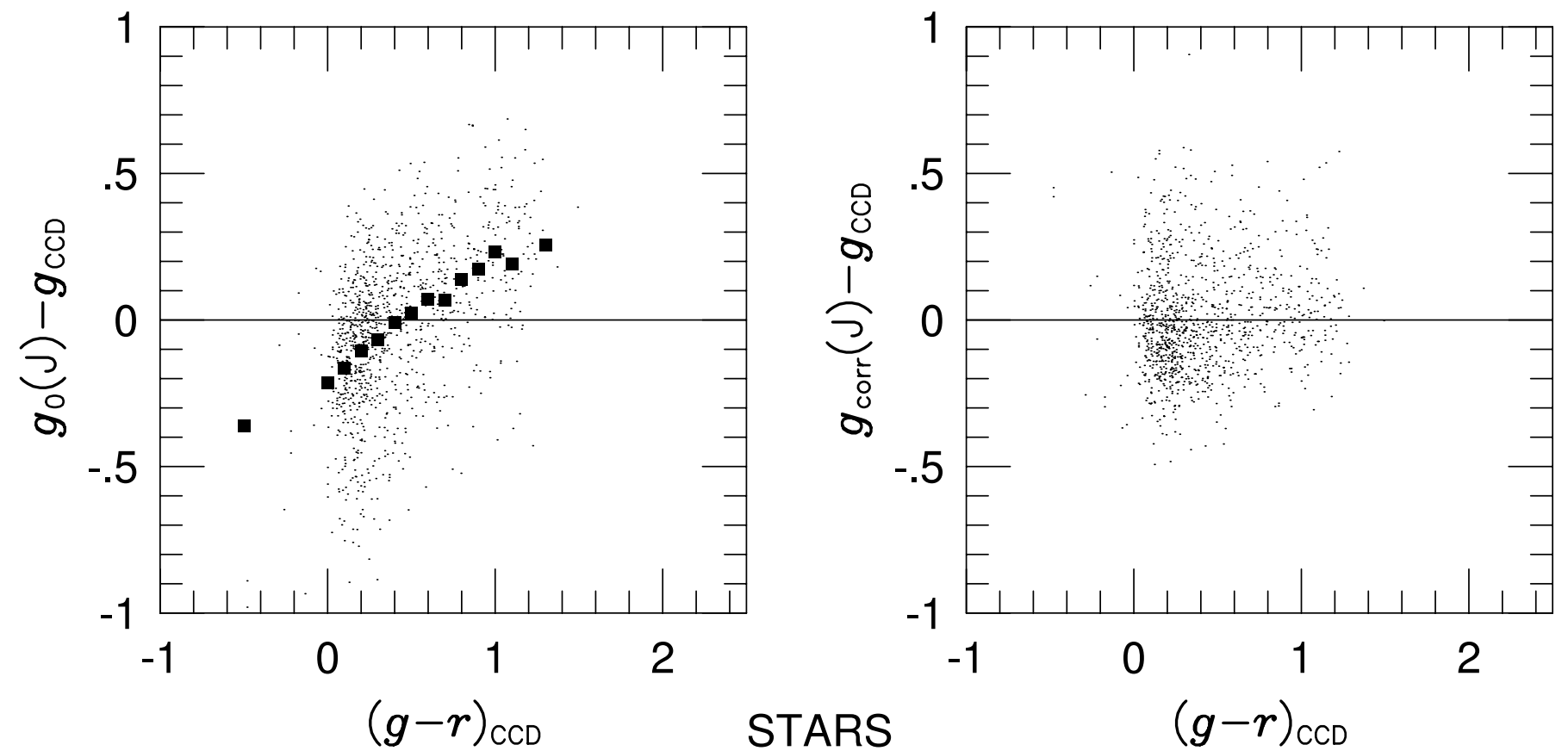

FIG. 9.-Difference between CCD and calibrated plate magnitudes, $g_{0}(J)-g_{\mathrm{CCD}}$, as a function of $g-r$ color, for galaxies (top) and stars (bottom), before and after a color correction has been applied. The filled squares show the median points used to derive the color correction. 

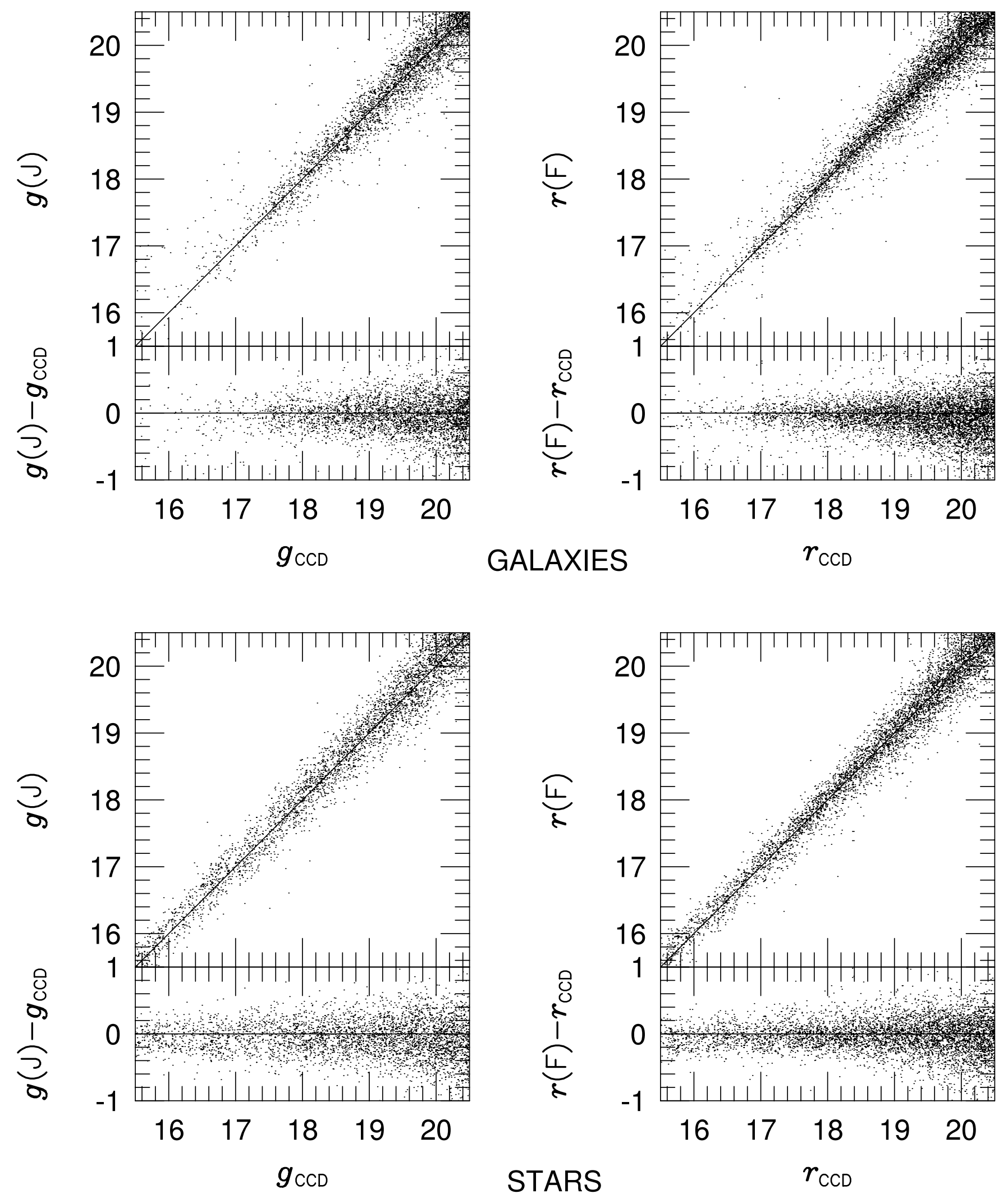

FIG. 10.-Comparison of the DPOSS $g$ and $r$ magnitudes derived from the transformation shown in Fig. 5 vs. the corresponding CCD magnitudes, for galaxies (top) and stars (bottom). 
TABLE 1

Total Photometric Errors

\begin{tabular}{|c|c|c|c|c|c|c|}
\hline \multirow[b]{2}{*}{$\mathrm{M}_{\mathrm{AG}}$} & \multicolumn{2}{|c|}{$g$} & \multicolumn{2}{|c|}{$r$} & \multicolumn{2}{|c|}{$i$} \\
\hline & Gal. & Star & Gal. & Star & Gal. & Star \\
\hline $17.0 \ldots \ldots \ldots \ldots \ldots . .$. & 0.25 & 0.22 & 0.13 & 0.15 & 0.17 & 0.22 \\
\hline 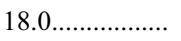 & 0.17 & 0.24 & 0.16 & 0.17 & 0.24 & 0.25 \\
\hline 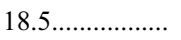 & 0.19 & 0.25 & 0.17 & 0.22 & 0.29 & 0.32 \\
\hline 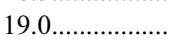 & 0.21 & 0.24 & 0.19 & 0.21 & 0.32 & 0.31 \\
\hline 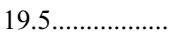 & 0.25 & 0.26 & 0.24 & 0.24 & 0.36 & 0.34 \\
\hline 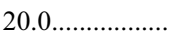 & 0.29 & 0.29 & 0.29 & 0.27 & 0.44 & 0.35 \\
\hline $20.5 \ldots \ldots \ldots \ldots \ldots . . .$. & 0.37 & 0.32 & 0.34 & 0.33 & 0.42 & 0.39 \\
\hline
\end{tabular}

CCD sequences, star-galaxy separation errors, inhomogeneities within individual plates, and errors in transforming all the plates to the fiducial instrumental system. Although the scatter is larger than those typically reported in the literature, it is the true combination of all possible errors. There are no additional effects to model or theoretical considerations to account for. These errors can therefore be used in any future work with no modification.

\subsection{Plate Overlap Tests}

The second test of photometric accuracy is a comparison of calibrated magnitudes from the overlap regions of two or more plates. This test is especially useful for examining photometric pistoning between the individually calibrated plates. A typical comparison in the $r$ band is shown in Figure 12. The results of this test show that for well-calibrated plates (those with more than 1000 calibration galaxies), the mean plate-toplate zero-point offset is zero, with a $1 \sigma$ scatter of $0.07 \mathrm{mag}$ in both the $g$ and $r$ bands, with only a slight magnitude dependence, as shown in Table 2. A few plate overlaps show much larger offsets (up to $\sim 0.2 \mathrm{mag}$ ); these indicate photometric errors due to sensitivity gradients across some individual plates. Such plates constitute $\sim 5 \%$ of the overall sample. Calibration in the $i$ band is significantly worse, with much larger sensitivity variations both within and between plates and many fewer calibrating objects.

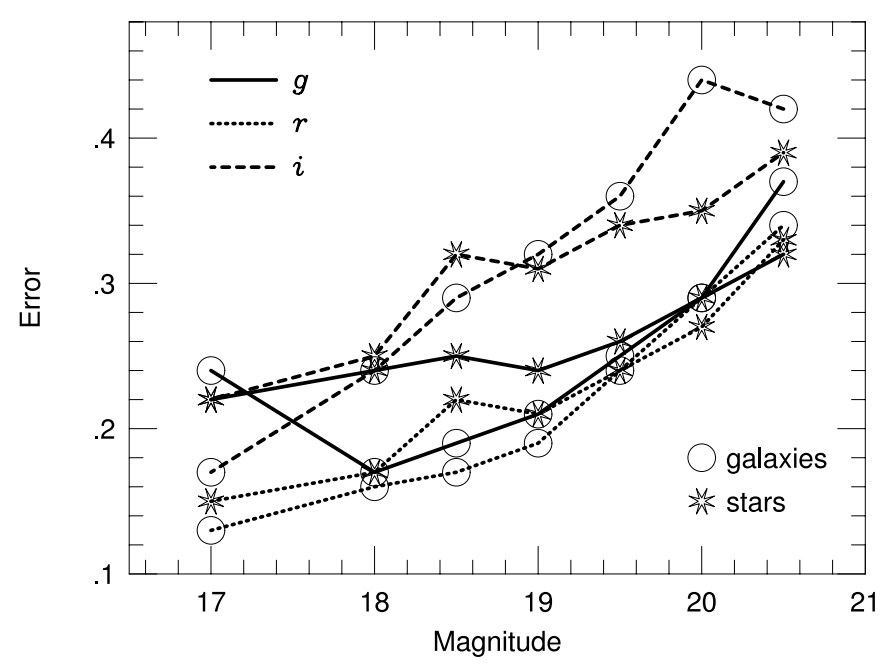

FIG. 11.-Total photometric errors as a function of magnitude, for stars (stars) and galaxies (circles), in the $g$ (solid lines), $r$ (dotted lines), and $i$ bands (dashed lines).

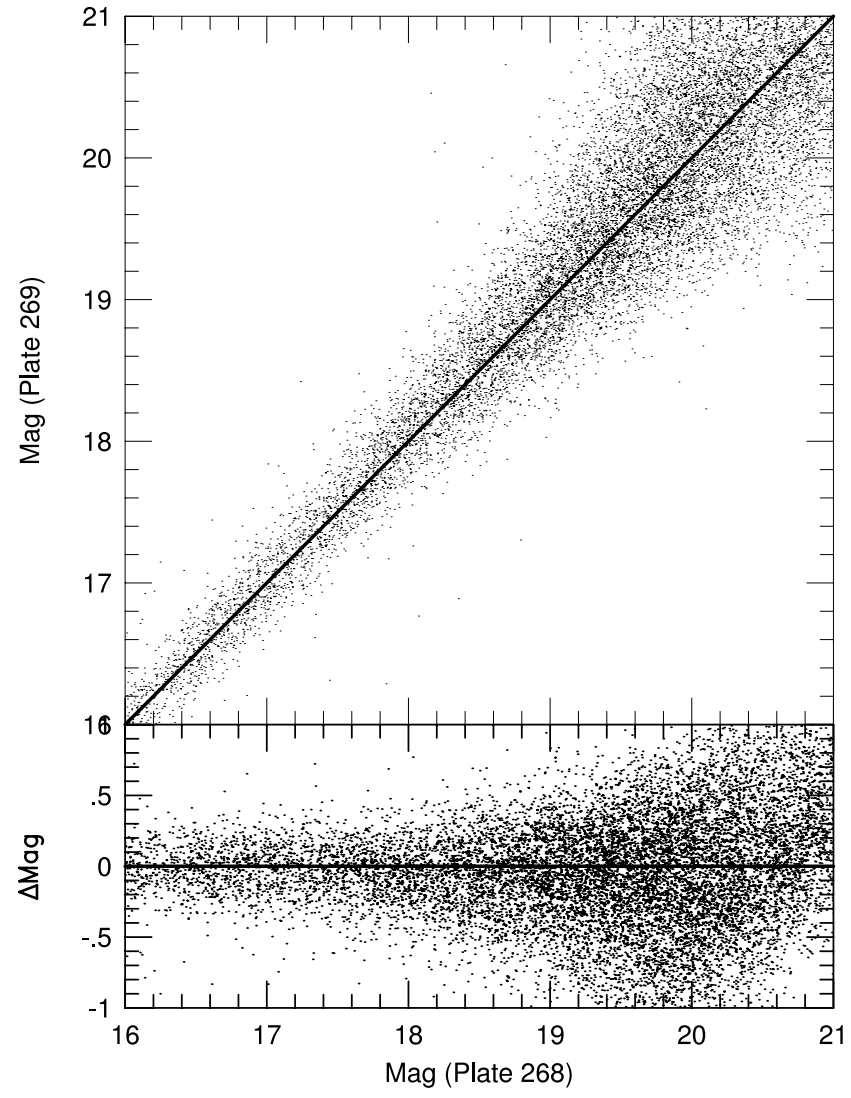

FIG. 12.-Comparison of $r$ calibrated magnitudes from the overlap regions of plates 268 and 269 .

The distributions of plate-to-plate zero-point offsets are well approximated by Gaussians with mean of 0 and standard deviations from Table 2, as shown in Figure 13. Therefore, users of DPOSS data may generate simulations including plate pistoning using the values provided in Table 2 .

In addition, we performed the same test as with the CCD data, using the plate overlap regions. We examined magnitude differences for all pairs of matched objects for plate pairs, in the same magnitude bins as with the plate versus CCD test. We then assumed that the measured photometric uncertainties are due to equal contributions from both plates and therefore divided the results by $\sqrt{2}$. The photometric uncertainties associated with individual objects, estimated in this way, are in complete agreement (to better than $10 \%$, or $0.01 \mathrm{mag}$ ) with the tests comparing the plate and CCD data. This is expected,

TABLE 2

Plate-to-Plate Offsets

\begin{tabular}{|c|c|c|c|}
\hline \multirow[b]{2}{*}{$\mathrm{M}_{\mathrm{AG}}$} & \multicolumn{3}{|c|}{$1 \sigma$ Scatter } \\
\hline & $g$ & $r$ & $i$ \\
\hline 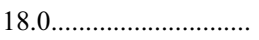 & 0.058 & 0.066 & 0.14 \\
\hline $18.4 \ldots \ldots \ldots \ldots$ & 0.059 & 0.070 & 0.14 \\
\hline $18.8 \ldots \ldots \ldots \ldots \ldots$ & 0.065 & 0.070 & 0.16 \\
\hline $19.2 \ldots \ldots \ldots \ldots \ldots \ldots$ & 0.071 & 0.073 & 0.13 \\
\hline 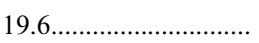 & 0.075 & 0.076 & 0.12 \\
\hline $20.0 \ldots \ldots \ldots \ldots \ldots \ldots$ & 0.080 & 0.078 & 0.13 \\
\hline 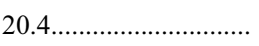 & 0.078 & 0.075 & 0.23 \\
\hline
\end{tabular}




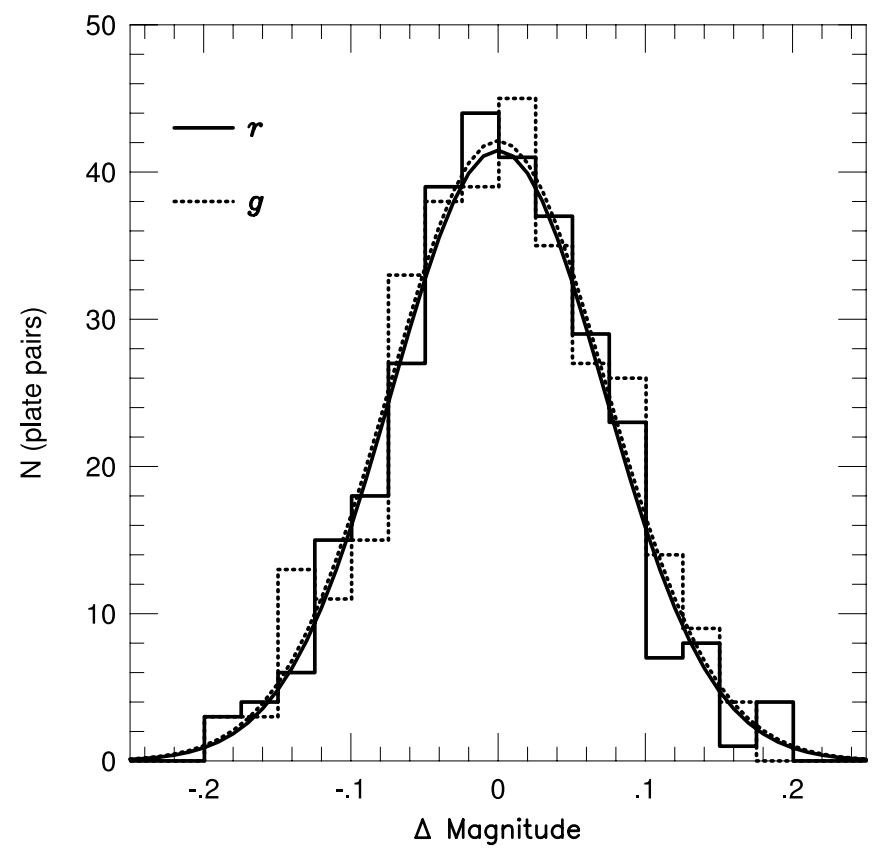

FIG. 13.-Distribution of plate-to-plate zero-point offsets at $m=19.4$ in the $r$ (solid line) and $g$ bands (dotted line). Gaussians with zero mean and $\sigma_{g, r}$ taken from Table 2 provide a good representation of the data.

as the errors in the plate-to-CCD comparison are dominated by the poorer plate photometry. We again conclude that the photometric uncertainties stated in Table 1 are robust.

\section{CONCLUSIONS}

We have presented the photometric calibration technique employed in DPOSS. Because of the large plate overlaps in the POSS-II and vast quantities of CCD calibration, we are able to perform accurate calibration over the entire high Galactic latitude northern sky. Our technique avoids significant systematic plate-to-plate zero-point offsets, resulting in stable photometry over extremely large angles. We provide the reader with robust photometric error measurements, which can be used during scientific analysis of the data. Our results are consistent with the plate-to-plate zero-point errors found by Weir et al. (1995a), although their sample was much smaller.

The resulting photometric catalogs, along with star/galaxy classifications (Odewahn et al. 2004), can be used for a large variety of astronomical projects that require large sky coverage. These include the search for $z>4$ quasars (Kennefick et al. 1995), unusual quasars (Brunner et al. 2003), the Northern Sky Optical Cluster Survey (Gal et al. 2000b, 2003), searches for small groups (Iovino et al. 2003), galaxy number counts, correlation functions, studies of galactic structure, and many more. $^{6}$

We thank the Norris Foundation for their generous support of the DPOSS project. R. R. G. was supported in part by an NSF Fellowship, NASA GSRP NGT5-50215, and a Kingsley Fellowship. This work would have been impossible without the POSS-II photographic team and the STScI digitization team. We also thank the Palomar TAC and directors for generous time allocations for the DPOSS calibration effort. Numerous past and present Caltech undergraduates (V. Desai, V. Hradecky, J. Meltzer, B. Stalder, J. Hagan, R. Stob, J. Kollmeier, and B. Granett) assisted in the taking of the data used in this paper. This work was made possible in part through the NPACI-sponsored Digital Sky project and a generous equipment grant from Sun Microsystems. Access to the POSS-II image data stored on the HPSS, located at the California Institute of Technology, was provided by the Center for Advanced Computing Research. The SDSC has generously allowed placement of our CCD data into their SRB as part of the National Virtual Observatory initiative.

\footnotetext{
6 The catalog data from DPOSS is at http://www.dposs.caltech.edu. In addition, the processed CCD imaging data used for calibration is available via the San Diego Supercomputing Center's Storage Resource Broker (SRB); directions for downloading this data are also at the DPOSS Web site.
}

Abell, G. O., Corwin, H. G., \& Olowin, R. P. 1989, ApJS, 70, 1

Brunner, R. J., et al. 2003, AJ, 126, 53

Djorgovski, S. G., de Carvalho, R. R., Gal, R. R., Odewahn, S. C., Mahabal, A. A., Brunner, R., Lopes, P. A. A., \& Kohl Moreira, J. L. 2003, Bol. Soc. Astron. Brasilìera, 23, 197

Djorgovski, S. G., Gal, R. R., Odewahn, S. C., de Carvalho, R. R., Brunner, R., Longo, G., \& Scaramella, R. 1998, in Wide Field Surveys in Cosmology, ed. S. Colombi, Y. Mellier, \& B. Raban (Gif-sur-Yvette: Ed. Frontières), 89

Gal, R. R., de Carvalho, R. R., Brunner, R., Odewahn, S. C., \& Djorgovski, S. G. 2000a, AJ, 120, 540

Gal, R. R., de Carvalho, R. R., Lopes, P. A. A., Djorgovski, S. G., Brunner, R. J., Mahabal, A., \& Odewahn, S. C. 2003, AJ, 125, 2064

Gal, R. R., de Carvalho, R. R., Odewahn, S. C., Djorgovski, S. G., \& Margoniner, V. E. 2000b, AJ, 119, 12

Groth, E. J., \& Peebles, P. J. E. 1986, ApJ, 310, 499

Iovino, A., de Carvalho, R. R., Gal, R. R., Odewahn, S. C., Lopes, P. A. A., Mahabal, A., \& Djorgovski, S. G. 2003, AJ, 125, 1660

Jarvis, J. F., \& Tyson, J. A. 1981, AJ, 86, 476

Kennefick, J. D., Djorgovski, S. G., \& de Carvalho, R. R. 1995, AJ, 110, 2553

Kent, S. M. 1985, PASP, 97, 165

Lasker, B. M., Doggett, J., McLean, B., Sturch, C., Djorgovski, S., de Carvalho, R. R., \& Reid, I. N. 1996, in ASP Conf. Ser. 101, Astronomical Data Analysis

\section{REFERENCES}

Software and Systems V, ed. G. H. Jacoby \& J. Barnes (San Francisco: ASP), 88

Maddox, S. J., Efstathiou, G., \& Sutherland, W. J. 1990, MNRAS, 246, 433 (MES)

McLean, B., Lasker, B., \& Lattanzi, M. 1998, BAAS, 192, No. 55.10

Monet, D. G. 1998, BAAS, 193, No. 120.03

Odewahn, S. C., \& Aldering, G. 1995, AJ, 110, 2009 (OA95)

Odewahn, S. C., de Carvalho, R. R., Gal, R. R., Djorgovski, S. G., Mahabal, A., \& Stalder, B. 2004, AJ, 128, 3092

Picard, A. 1991, Ph.D. thesis, Caltech

Reid, I. N., et al. 1991, PASP, 103, 661

Schneider, D. P., Gunn, J. E., \& Hoessel, J. G. 1983, ApJ, 264, 337

Thuan, T. X., \& Gunn, J. E. 1976, PASP, 88, 543

Tody, D. 1986, Proc. SPIE, 627, 733

Valdes, F. 1982, Proc. SPIE, 331, 465

Wade, R. A., Hoessel, J. G., Elias, J. H., \& Huchra, J. P. 1979, PASP, 91, 35

Weir, N., Djorgovski, S., \& Fayyad, U. M. 1995a, AJ, 110, 1

Weir, N., Fayyad, U. M., \& Djorgovski, S. 1995b, AJ, 109, 2401

Weir, N., Fayyad, U. M., Djorgovski, S. G., \& Roden, J. 1995c, PASP, 107, 1243

York, D. G., et al. 2000, AJ, 120, 1579 